\title{
Optical and Morphological Properties of Carbon Nanotube-Anatase Nanocomposites: Improvements in Visible Absorbance
}

\author{
Marianna Barberio $^{1,2^{*}}$, P. Barone ${ }^{1,2}$, A. Imbrogno ${ }^{1}$, A. Bonanno ${ }^{1}, \mathrm{~F}_{\mathrm{X}} \mathrm{Xu}^{1}$ \\ ${ }^{1}$ Dipartimento di Fisica, Università della Calabria, Cosenza, Italy; ${ }^{2}$ Dipartimento di Biologia, Ecologia e Scienze della Terra, Uni- \\ versità della Calabria, Cosenza, Italy. \\ Email: * marianna.barberio@fis.unical.it
}

Received July 21 ${ }^{\text {st }}$, 2013; revised August 21 ${ }^{\text {st }}, 2013$; accepted August $28^{\text {th }}$, 2013

Copyright (c) 2014 Marianna Barberio et al. This is an open access article distributed under the Creative Commons Attribution License, which permits unrestricted use, distribution, and reproduction in any medium, provided the original work is properly cited. In accordance of the Creative Commons Attribution License all Copyrights @ 2014 are reserved for SCIRP and the owner of the intellectual property Marianna Barberio et al. All Copyright (C) 2014 are guarded by law and by SCIRP as a guardian.

\begin{abstract}
In this work, we present the growth and optical characterization of carbon nanotube/ $\mathrm{TiO}_{2}$ anatase nanocomposites. The composites are obtained by doping a bulk of anatase nanometric grains with different weight percentages of carbon nanotube (from $0.1 \%$ to $50 \%$ ). We observe that only for tube concentrations between $0.1 \%$ and $\mathbf{2 \%}$, the growth process shows the formation of a matrix where carbon nanotubes are uniformed and dispersed in a bulk of $\mathrm{TiO}_{2}$ grains. Moreover, the X-ray photoelectron spectroscopy and surface morphology analysis (conducted from AFM images) indicated that the CNT absorption is a simple physisorption without chemical bonds formations between tube and dioxide. Finally, absorption in all the visible range has the increase of about $60 \%$ at very low concentration ( $2 \%)$ of carbon nanotubes.
\end{abstract}

\section{KEYWORDS:}

\section{Carbon Nanotubes; Anatase; Nanocomposites; Visible Absorbance}

\section{Introduction}

Recently the interest of scientific community has been aimed to study nanostructured materials for their potential application in photovoltaic, electro-optical, micromechanical and sensor devices [1-3].

Particularly, nonporous $\mathrm{TiO}_{2}$ thin films for dyesensitized and ETA (Extremely Thin Absorber) solar cells have been under intensive study for many years [2,3]. In fact, $\mathrm{TiO}_{2}$, usually used in both mineral phases (anatase and rutile), supplies several advantages due to its high chemical stability, excellent functionality, nontoxicity, optical proprieties (both are transparent in the visible and absorbed in the near ultraviolet region [3]) and relatively low cost.

But, $\mathrm{TiO}_{2}$ has the serious disadvantage that is used in the photocatalytical process only for the UV light [4-8]. In fact, only the $3 \%$ of the solar spectrum can be utilized

\footnotetext{
${ }^{*}$ Corresponding author.
}

due to its wide intrinsic band gap. It is so of great interest to find ways for extending the absorption wavelength range of $\mathrm{TiO}_{2}$ within the visible region without decreasing its photocatalytic activity. Carbon-doped titanium dioxide has been selected as best candidate, because theoretical and experimental studies [8-12] have demonstrated the possibility to reduce drastically the band gap of $\mathrm{TiO}_{2}$, improving the adsorption properties of dioxide in the visible range. Moreover, C-doped $\mathrm{TiO}_{2}$ possesses much higher photocatalytic activity than the non-doped one [10-12]. Currently there are two different techniques for $\mathrm{C}$ doping. The inner approach is using $\mathrm{TiC}$ and annealing it at higher temperature to $98 \%$, and the $\mathrm{TiO}_{2}$ conversion or the outer approach is using amorphous $\mathrm{TiO}_{2}$ and doping it with carbon from precursor source like glucose [13].

In this work, we present an alternative C-doping using carbon nanotube (CNT). Recently efforts to combine anatase with carbon nanotubes in simple mixtures or nano- 
composite materials have been made to create more highly reactive photocatalytic materials [5-7]. Moreover, we proposed in recent works $[14,15]$ a technique to make CNT/ anatase micro-composites and $\mathrm{TiO}_{2}$ /rutile nanocomposites [16] using a simple chemical-mix method. In first case, the produced samples appear as grains of anatase bulk, with dimensions ranging between few and 50 - 60 microns with the CNTs wrapped around these grains. While for rutile nanocomposites, we observe that the carbon nanotubes are distributed in the interstitial between rutile grains. In both cases, the luminescence emission of these composites is strongly modified with respect to pure dioxide with a strong reduction in the visible region [15] while the optical absorbance in visible range increases to about 6\% [14-16] indicating significative changes in optical band.

In this work, we make the use of the growth technique in $[15,16]$ to obtain nanocomposites of anatase grains doped with carbon nanotubes to improve the properties of optical absorbance in visible ranges for anatase structure also already obtained for rutile. We develop composites with different CNT weight percentages to obtain composites with morphological and optical properties optimized. The characterization of sample properties was

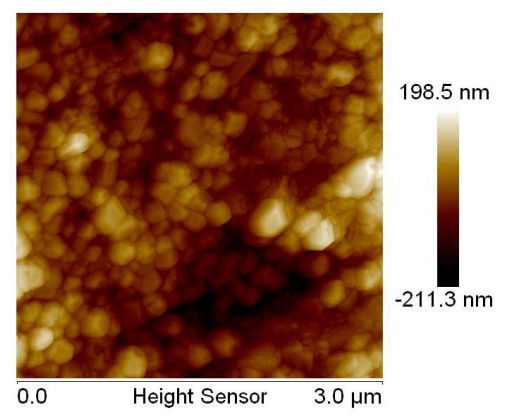

(a)

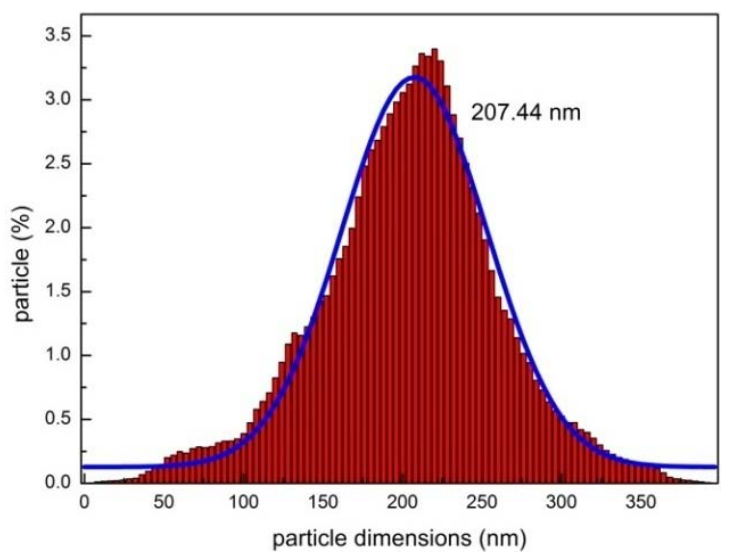

(c) taken by X-ray photoelectron spectroscopy, SEM and AFM (associated by the study of surface roughness and fractal dimensions) and by direct measurements of optical absorbance.

\section{Experiments}

\subsection{Materials}

The CNT powder used in this study was purchased from "Arry-nano" (Germany), with a nominal purity greater than $95 \%$, an average diameter of about $15 \mathrm{~nm}$ and a length of 5 - $30 \mathrm{~mm}$.

Nanometric anatase particles were prepared by a solgel method. AFM images of grains in Figure 1(a) show a regular surface composed by aggregates of grains. The histogram of grain diameter distribution of anatase is shown in Figure 1(c) (elaborated by Nanoscope software); we obtain, by Gaussian fitting curve, an average diameter of about $200 \mathrm{~nm}$ with a distribution varying from 50 to $300 \mathrm{~nm}$.

$\mathrm{CNTs} / \mathrm{TiO}_{2}$ composites were prepared using a simple mixing method [14-18] with a sequence of mixing and heating processes. First, CNTs and $\mathrm{TiO}_{2}$ powders were dispersed in diclorobenzene and sonicated for $1.5 \mathrm{~h}$. So,

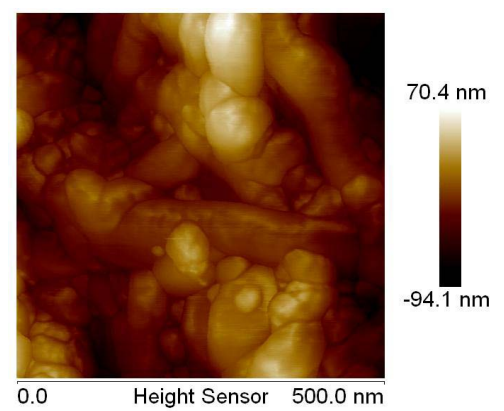

(b)

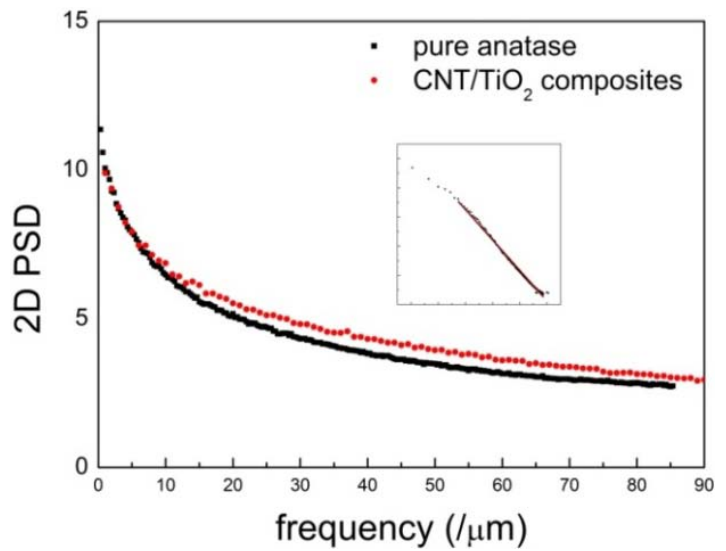

(d)

Figure 1. AFM images of pure $\mathrm{TiO}_{2}$ samples (a) and composites obtained with $2 \%$ of CNT (b). Diameter distribution of anatase grains (c) and 2D PSD (d) for pure anatase and nanocomposites with CNT $2 \%$, evaluated from software Nanoscope from Bruker (Inset in D: log-log plot of 2D PSD and linear fit for evaluation of fractal dimensions). 
the suspension was deposited on a clean copper plate (2 $\mathrm{cm} \times 2 \mathrm{~cm}$ in size) forming a thin film of about $500 \mathrm{~mm}$, then heated to $180^{\circ} \mathrm{C}$ in air on a hot plate for 15 minutes to facilitate solvent evaporation (further details on sample preparations were discussed in [18]). The composites were prepared with different CNT weight concentrations (0.1\%, $0.2 \%, 0.6 \%, 1 \%, 1.5 \%, 2 \%, 10 \%, 20 \%, 25 \%$ and $50 \%)$.

\subsection{Methods}

Chemical, morphological and optical properties of our samples have been obtained by surface spectroscopy and microscopy. X-ray photoelectron spectroscopy reveals the chemical properties of surface (both elemental and bonding information) while SEM and AFM microscopy showed the sample morphology. Finally, the optical properties were obtained measuring directly the visible optical absorbance.

XPS measurements were conducted in a UHV chamber equipped for standard surface analysis with a base pressure in the range of low $10^{-9}$ torr. Non monochromatic Mg-Ka X-ray ( $h n=1253.64 \mathrm{eV})$ was used as excitation source. The XPS spectra were calibrated with the C1s peak of a pure carbon sample (binding energy 284.6 $\mathrm{eV})$.

Optical absorbance measurements were taken with an Olympus microscope (Horiba-Jobyn Yvon) mounting objectives of $10 \times, 50 \times$ and $100 \times$ magnification and equipped with a white lamp and a Triax 320 (Horiba-Jobyn-Yvon) spectrometer working in the $200-1500 \mathrm{~nm}$ range.

We measured directly the reflected spectrum $\left(I_{r}(\lambda)\right)$ and obtained, by assuming null the transmittance for bulk samples, the reflectance $(r(\lambda))$ and the absorbance $(\varepsilon(\lambda))$ as a function of wavelength by the relation:

$$
\begin{gathered}
r(\lambda)=\frac{I_{r}(\lambda)}{I_{s}(\lambda)} \\
\varepsilon(\lambda)=1-r(\lambda)
\end{gathered}
$$

where $I_{s}(\lambda)$ is the source spectrum. Total absorbance was obtained by integration of $\varepsilon(\lambda)$.

The AFM images are taken by an Icon Bruker AFM working in tapping mode. The images have a resolution of $512 \times 512$ lines and are acquired with scanning rate of about $1 \mathrm{~Hz}$. The obtained images were processed with the Nanoscope software (provided by Bruker) to evaluate size distribution of particle dimensions, the roughness, the 2D isometric power spectral density (PSD) and the relative power (obtained as Integral of PSD). It is, in fact, recognized that the surface morphology and dynamics of growing surfaces processes obey simple scaling laws associated with roughness, fractal dimensions and surface power ([17-19] and references therein). Indeed, the changes in surface morphology are strictly related to changes in these three parameters, which are, so, good indices for representing the nano and micro structures of surfaces.

Fractals, in particular, retain properties which can reveal the surface properties. In fact, they reveal details on arbitrarily small scales which exhibiting a fractal dimension $D_{f}$ which characterizes the topological dimensions of analyzed surfaces (if $D_{f}$ is a number from 2 to 3 the fractal set fills the space where it is embedded).

The fractal Dimensions for each substrate were determined as $D_{f}=(8-\beta) / 2$, where $\mathrm{b}$ is the slope of the $2 \mathrm{D}$ PSD calculated in a log plot as explained in [17].

The SEM images were performed using a Cambridge Stereoscan SEM with a $20 \mathrm{keV}$ electron beam and a current of $3.4 \mu \mathrm{A}$.

\section{Results and Discussion}

SEM images of composites in Figure 2 shows a very different behavior for different CNT concentration. Composites obtained with high concentration of CNT $(20 \%$ and 10\% in Figures 2(a)-(d)) appears as a bulk of carbon nanotube in which aggregates of anatase grains are randomly overlapped (the aggregates are clearly visible in images taken in back scattered mode, Figures 2(b) and (d), while the tube are observable in these obtained in secondary electron mode, Figures 2(a) and (c)). For lower CNT concentration, as visible in Figure 2(e), the composites appears as a uniform bulk of $\mathrm{TiO}_{2}$ grains and the nanotube are dispersed in the interstices between aggregates. This behavior is confirmed by AFM images in Figure 1(b). It is clear that the tube is intercalated between the grains. So we can conclude that only with low percentage of CNT we realize a homogeneous composite where $\mathrm{CNT}$ and $\mathrm{TiO}_{2}$ are perfectly integrated in a unique matrix.

Morphologic surface properties of composites with low CNT concentrations are substantially unchanged between pure $\mathrm{TiO}_{2}$ grains and $\mathrm{CNT} / \mathrm{TiO}_{2}$ composites. The surface roughness, obtained by Nanoscope software, passes from $56.3 \mathrm{~nm}$ of pure sample to $51.3 \mathrm{~nm}$ of composites while surface roughness and fractal dimensions, evaluated by 2D Isotropic PSD in Figure 1(d), varies from $0.051 \mathrm{~nm}^{2}$ to $0.057 \mathrm{~nm}^{2}$ and 1.92 to 2.07 respectively. These very low changes in surface morphology parameters suggest that the growth of composite with low weight percentage of CNT correspond to a doping process of $\mathrm{TiO}_{2}$ confirming the results presented in our previous work for nanometric rutile [16].

For all samples, XPS data (Figure 3 for the composite with CNT $2 \%$ as example for all samples) indicate the absence of chemical bonds between carbon nanotubes and metals specifying the Van deer Waals nature of 


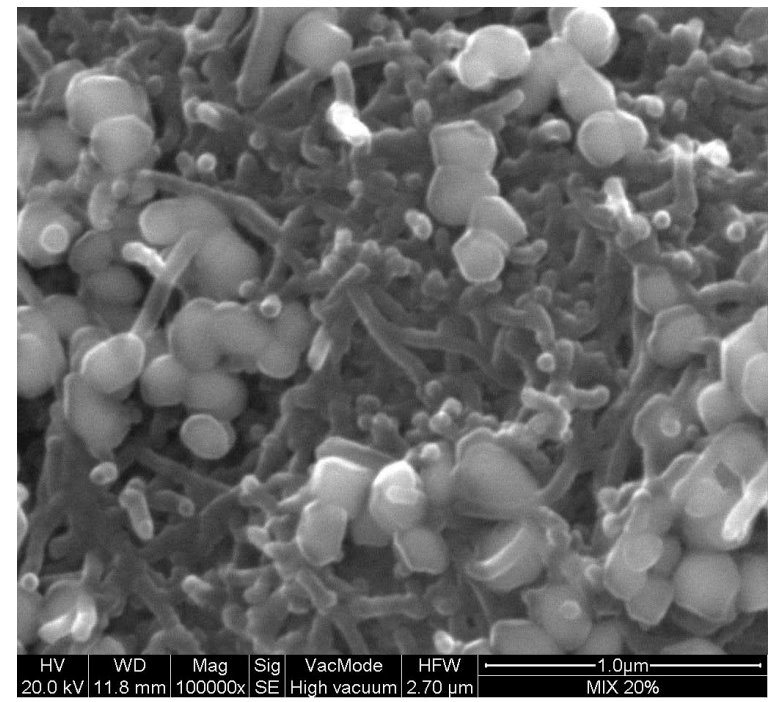

(a)

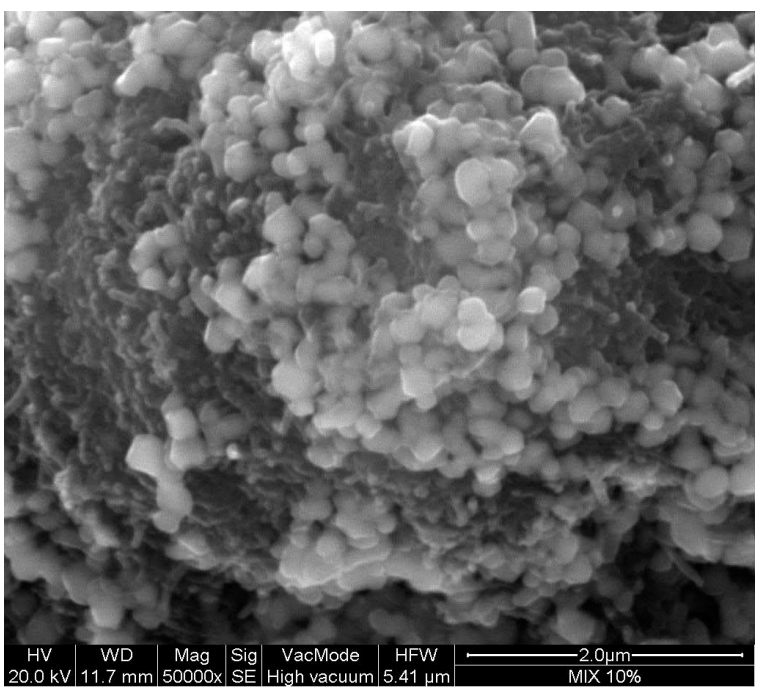

(c)

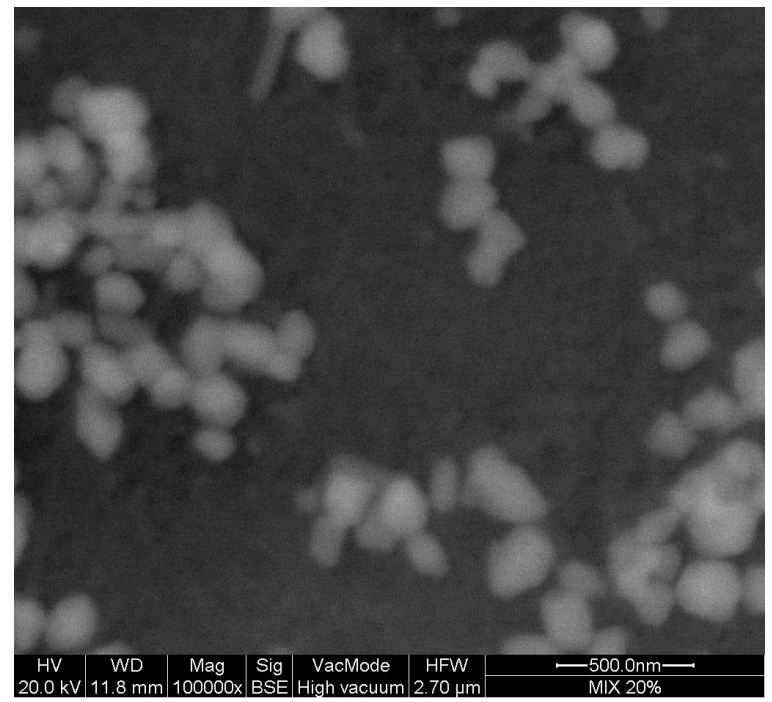

(b)

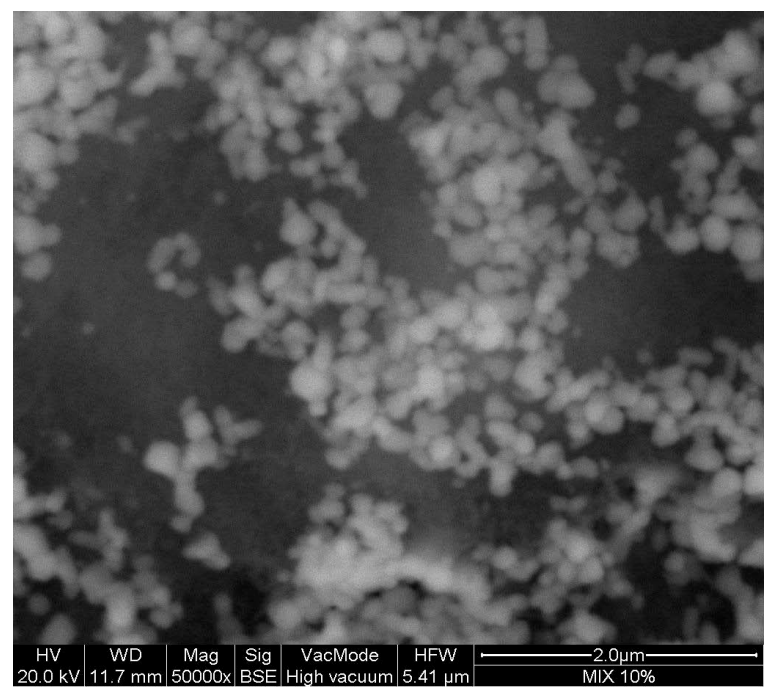

(d)

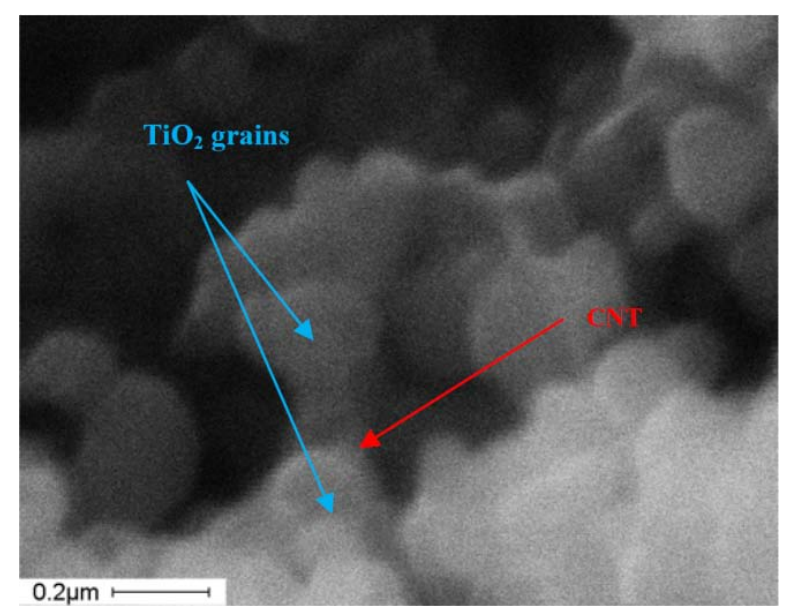

(e)

Figure 2. SEM images of samples obtained with $20 \%$ of CNT ((a) SE-SEM and (b) BSE-SEM), CNT 10\% ((c) SE-SEM and (d) BSE-SEM) and CNT $2 \%(e)$. 
bonding between particle and tubes and the physisorption character of particle absorption on sheet surface. In fact, the survey spectra (Figure 3(A)) indicated in all sample the only presence of Carbon, Oxygen (also the carbon nanotubes are rich in oxygen which can be eliminate only after heating at very high temperatures [20]) and Titanium without presence of surface impurities. The main XPS lines for all elements (Figures 3(B)-(D)) are these typical of carbon nanotubes and of Titanium dioxide. The C1s line (3C) exhibits three lines at $284 \mathrm{eV}$ (C main line indicating C-C bonding), $287 \mathrm{eV}$ (C-O bonds) and 303 $\mathrm{eV}$ which is due to plasma loss in carbon nanotubes. The Ti $2 p$ line (3D) is structured and exhibits two main structures (evidenced by a Gaussian analysis of the line), at 457 and $462 \mathrm{eV}$ and a satellites structured band with a peak at about $20 \mathrm{eV}(477 \mathrm{eV})$. The main band at $457 \mathrm{eV}$ can attributed to $\mathrm{Ti} 2 \mathrm{p}_{3 / 2}$ in $\mathrm{TiO}_{2}$, while the structure after about $5 \mathrm{eV}$ (at $462 \mathrm{eV}$ ) is ascribed to $\mathrm{Ti} 2 \mathrm{p}_{1 / 2}$ in $\mathrm{TiO}_{2}$ (the doublet separation of $\sim 5 \mathrm{eV}$ between the $2 \mathrm{p}_{1 / 2}$ and $2 \mathrm{p}_{3 / 2}$ peaks is characteristic of $\left.\mathrm{TiO}_{2}\right)[16,20,21]$. The assignment of satellites lines in titanium dioxide is not without ambiguity and involves two shake-up physical processes (charge transfer excitations) or energy losses. The group of satellite peaks, involving a wide band at $12 \mathrm{eV}$ from

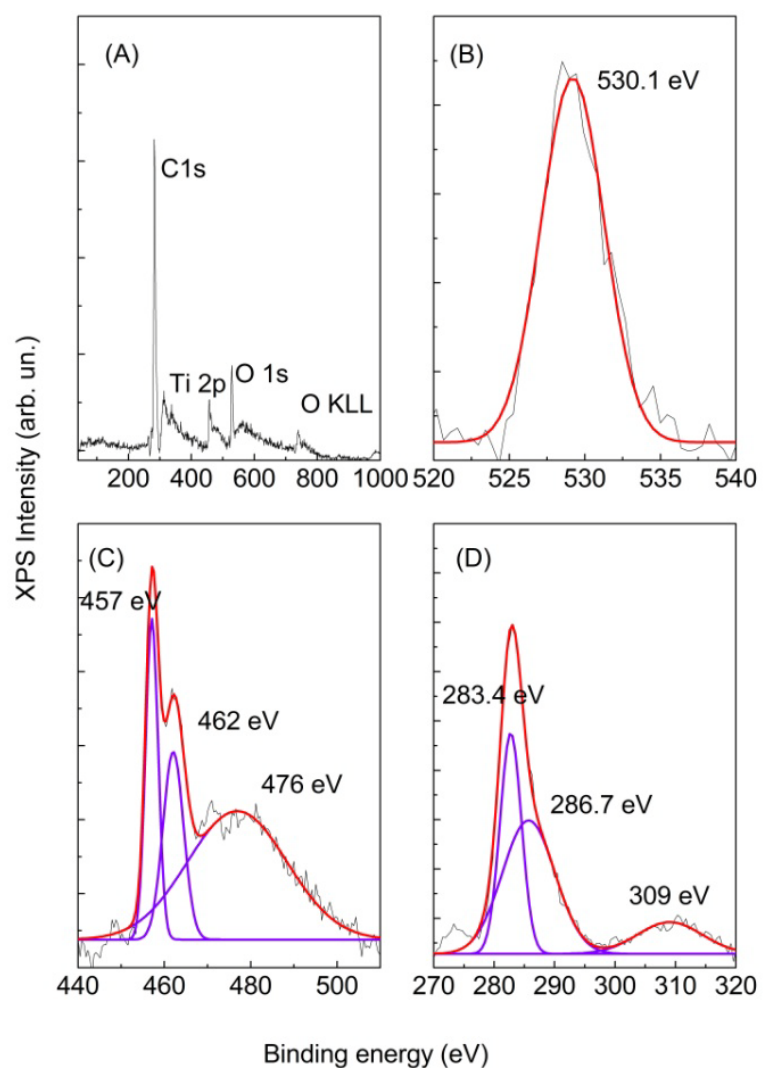

Figure 3. XPS data for CNT (2\%)/anatase composites as example of all composites: survey spectra (A), $O$ 1s line (B), C 1s line (C), Ti 2p line (D). the main band on the higher energies side, would be related to transitions of valence electrons towards continuum states, while the peak observed at about $20 \mathrm{eV}$ could be attributed to transitions of valence electrons towards empty states at the top of the $4 \mathrm{~s}$ band $[21,22]$.

Therefore, all the Ti $2 p$ lines are those characteristics of $\mathrm{TiO}_{2}$ and the presence of carbon nanotubes do not involve changes in $\mathrm{Ti}$ spectral lines, confirming the absence of Ti-C chemical bonds.

The $\mathrm{O}$ 1s line (Figure 3(B)) presents a main structure at roughly $530 \mathrm{eV}$ that is usually assigned to oxygen bonded with tetravalent $\mathrm{Ti}^{3+}$ ions in $\mathrm{TiO}_{2}$ [19]. The presence of C-O chemical bonds in the spectra (demonstrated by the 285.7 line in C 1s) are due to chemical bonds between carbon and oxygen adsorbed in carbon nanotubes before the mixing with anatase particles and do not indicate peculiar anatase/CNT chemical bonds. In fact, the carbon nanotubes are pristine and contain a large amount of oxygen (typically of the order of $10 \%$ [12-15]).

The optical absorbance spectra in visible range are showed in Figure 4. All the samples (either with high and low CNT concentrations) show a wide absorption spectrum which cover all the visible range with a intensity which increases with CNT concentration. The total absorbance in visible range increases with CNT concentration (Figure 5) passing from $10 \%$ of pure $\mathrm{TiO}_{2}$ to $97 \%$

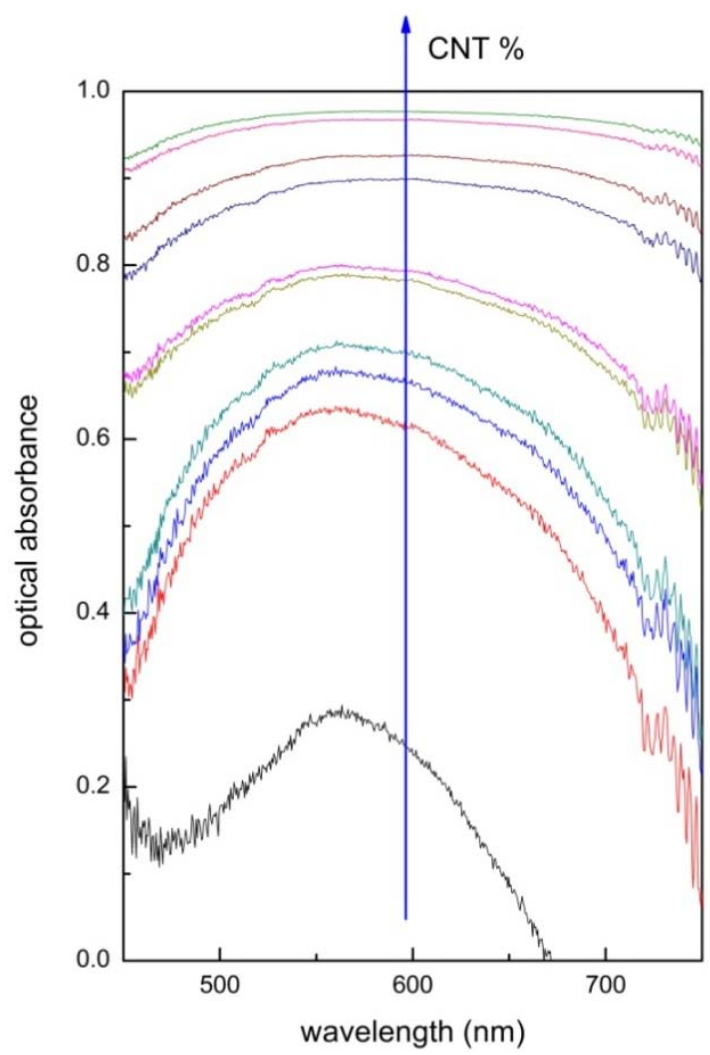

Figure 4. Optical absorbance spectra of all composites. 


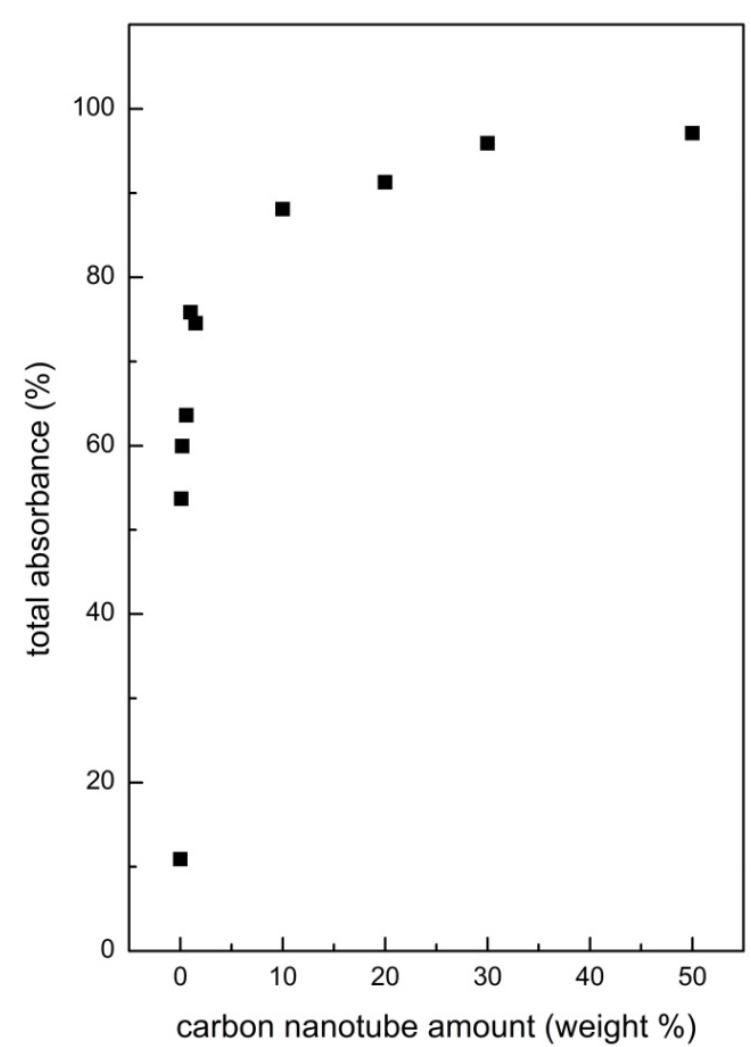

Figure 5. Total optical absorbance (\%) as function of CNT amount on composites.

of samples with $50 \%$ of CNT. In particular, the composites at low CNT concentration $(0.2 \%-2 \%)$ show an optical absorbance which varies between $60 \%$ and $80 \%$, with a variation respect to pure anatase of $60 \%$.

This enhancement in optical absorbance indicates the high effectiveness of doping with CNT respect to doping with simple $\mathrm{C}$ atoms. In fact, theoretical and experimental work on $\mathrm{C}$ doping indicating that usual C-doping needed a $5 \%$ concentration of $C$ to have significative variations in optical absorbance [10,11]. Moreover the improvements in optical absorbance obtained with these CNT/ anatase composites is much higher than these obtained from CNT/rutile composites in our previous work [16], where the absorbance of the composite respect to pure rutile increases only of $6 \%$. We attribute these differences in doping effectiveness in the very low absorbance of pure anatase respect to pure rutile and can conclude that the doping of CNT can lead to a very enhancement in optical properties of anatase and so in its photocatalitic and photovoltaic activity.

\section{Conclusions}

In this work, we have presented the results obtained in growth of $\mathrm{CNT} / \mathrm{TiO}_{2}$ anatase nanocomposites. We develop composites with different weight percentages of car- bon nanotube obtaining an effective homogeneous and uniform matrix of CNT/anatase only for CNT percentage between $0.1 \%$ and $2 \%$. Composites with CNT concentrations higher than $2 \%$ appear, in fact, at morphological analysis (conducted on SEM and AFM conditions) as a bulk of carbon nanotubes with micro-clusters of $\mathrm{TiO}_{2}$ grains randomly dispersed on surfaces. While at low CNT concentration, AFM and SEM images indicate the formation of a uniform bulk of anatase grains where the carbon nanotubes are inserted in the interstitials between grains. The growth process, as indicated by XPS and surfaces analysis, is a simple physisorption without sign of Ti-C chemical bonds. In fact, the XPS lines of $\mathrm{C}$, Ti and $\mathrm{O}$ are these of pure $\mathrm{TiO}_{2}$ and CNT and there aren't significative changes in roughness, power or fractal dimensions between pure anatase and nanocomposites.

Finally we observe great improvements in optical absorbance in visible range. The absorbance changes from $10 \%$ of pure anatase to $70 \%$ of composites with $1.5 \%$ of CNT indicating a very great effectiveness of CNT doping with respect to simple C-doping and CNT doping of rutile opening the possibility to integrate these composites in photocatalic and photovoltaic devices of new generation.

\section{REFERENCES}

[1] M. Hemissi and H. Amardjia-Adnani, "Optical and Structural Properties of Titanium Oxide Thin Fils Prepared by Sol-Gel Method,” Digest Journal of Nanomaterials and Biostructures, Vol. 2, No. 4, 2007, pp. 299-305.

[2] J. Sabataityté, I. Oja, F. Lenzman, O. Volobujeva and A. Krunks, "Characterization of Nanoporous $\mathrm{TiO}_{2}$ Films Prepared by Sol-Gel Method,” Comptes Rendus Chimie, Vol. 9, No. 5-6, 2006, p. 708.

[3] R. Zallem and M. P. Moret, "The Optical Absorption Edge of Brookite $\mathrm{TiO}_{2}$," Solid State Communications, Vol. 137, No. 3, 2006, pp. 154-157.

http://dx.doi.org/10.1016/j.ssc.2005.10.024

[4] A. R. Ghande and J. O. Fernandez, “A Simple Method to Synthesize Light Active N-Doped Anatase $\left(\mathrm{TiO}_{2}\right)$ Photocatalyst," Bulletin of the Catalysis Society of India, Vol. 4, 2005, pp. 131-134.

[5] K. Woan, G. Pyrgiotakis and W. Sigmund, "Photocatalytic Carbon-Nanotube- $\mathrm{TiO}_{2}$ Composites," Advanced Materials, Vol. 21, No. 21, 2009, pp. 2233-2239. http://dx.doi.org/10.1002/adma.200802738

[6] Y. Yao, G. Li, S. Ciston, R. M. Lueptow and K. A. Gray, "Photoreactive $\mathrm{TiO}_{2} /$ Carbon Nanotube Composites: Synthesis and Reactivity," Environmental Science \& Technology, Vol. 42, No. 13, 2008, pp. 4952-4957. http://dx.doi.org/10.1021/es800191n

[7] R. I. Bickley, T. Gonzalezcarreno, J. S Lees and R. J. Tilley, "A Structural Investigation of Titanium Dioxide Photocatalysts,” Journal of Solid State Chemistry, Vol. 92, 
No. 1, 1991, pp. 178-190. http://dx.doi.org/10.1016/0022-4596(91)90255-G

[8] M. Kaneko, “Ohkura, Photocatalysis,” Springer, Tokyo, 2002.

[9] H. Kamisaka, T. Adachi and K. Yamashitaa, “Theoretical Study of the Structure and Optical Properties of CarbonDoped Rutile and Anatase Titanium Oxides,” The Journal of Chemical Physics, Vol. 123, No. 8, 2005, Article ID: 084704. http://dx.doi.org/10.1063/1.2007630

[10] W. Choi, A. Termin and M. R. Hoffmann, "The Role of Metal-Ion Dopants in Quantum-Sized $\mathrm{TiO}_{2}$ : Correlation Between Photoreactivity and Charge-Carrier Recombination Dynamics,” Journal of Physical Chemistry, Vol. 98, No. 51, 1994, pp. 13669-13679. http://dx.doi.org/10.1021/j100102a038

[11] H. Wang and J. P. Lewis, "Second-Generation Photocatalytic Materials: Anion-Doped $\mathrm{TiO}_{2}$," Journal of Physics: Condensed Matter, Vol. 18, No. 2, 2006, pp. 421434. http://dx.doi.org/10.1088/0953-8984/18/2/006

[12] J. Pascual, J. Camassel and H. Mathieu, "Fine Structure in the Intrinsic Absorption Edge of $\mathrm{TiO}_{2}$," Physical Review $B$, Vol. 18, No. 10, 1978, pp. 5605-5614. http://dx.doi.org/10.1103/PhysRevB.18.5606

[13] W. Ren and Z. Ai, "Low Temperature Preparation and Visible Light Photocatalytic Activity of Mesoporous Carbon-Doped Crystalline $\mathrm{TiO}_{2}$," Applied Catalysis B: Environmental, Vol. 69, No. 3-4, 2007, pp. 138-144. http://dx.doi.org/10.1016/j.apcatb.2006.06.015

[14] M. Barberio, P. Barone, A. Bonanno and A. Oliva, "Synthesis and Characterization of Carbon Nanotubes Wrapped on Anatase Microparticles," Particle and Particle Systems Characterization, Vol. 28, No. 3-4, 2012, pp. 64-70.

[15] M. Barberio, P. Barone, V. Pingitore and A. Bonanno, “Optical Properties of $\mathrm{TiO}_{2}$ Anatase-Carbon Nanotubes Composites Studied by Cathodoluminescence Spectroscopy,” Superlattice and Microstructure, Vol. 51, No. 1, 2012, pp. 177-183. http://dx.doi.org/10.1016/j.spmi.2011.11.011

[16] M. Barberio, P. Barone, A. Imbrogno, V. Pingitore, F. Xu and A. Bonanno, "Optical and Structural Properties of Carbon Nanotube-Rutile Heterostructures," Journal of Chemistry and Chemical Engineering, Vol. 6, 2012, pp. 199-208.

[17] F. Gentile, E. Battista, A. Accardo, M. L. Coluccio, M. Asande, G. Perrozziello, G. Das, C. Liberale, F. De Angelis, P. Candeloro, P. Decuzzi and E. Di Fabrizio, "Fractal Structure Can Explain the Increased Hydrophobicity of Nanoporous Silicon Films," Microelectronic Engineering, Vol. 88, No. 8, 2011, pp. 2537-2540. http://dx.doi.org/10.1016/j.mee.2011.01.046

[18] M. Saitou, W. Oshikawa and A. Makabe, "Characterization of Electrodeposited Nickel Film Surfaces Using Atomic Force Microscopy," Journal of Physics and Chemistry of Solids, Vol. 63, No. 9, 2002, pp. 1685-1689. http://dx.doi.org/10.1016/S0022-3697(01)00254-2

[19] A. M. Skolnik, W. C. Hughes and B. H. Augustine, “A Metallic Surface Corrosion Study in Aqueous NaCl Solutions Using Atomic Force Microscopy (AFM)," The Chemical Educator, Vol. 5, No. 1, 2000, pp. 8-13. http://dx.doi.org/10.1007/s00897990350a

[20] A. Bonanno, M. Barberio, P. Barone, M. Camarca, D. R. Grosso, R. Vasta, et al., "Changes in Electronic Properties of Carbon Structures by Evaporation and Implantation of Alkali Metals," Vacuum, Vol. 84, No. 8, 2010, pp. 1025-1028. http://dx.doi.org/10.1016/j.vacuum.2009.10.0 $\underline{39}$

[21] “NIST XPS Data Base.” http://srdata.nist.gov/xps/main_search_menu.aspx

[22] H. Chermette, P. Pertosa and F. M. Michel-Calendini, "Molecular Orbital Study of Satellites in XPS Spwctra of $\mathrm{BaTiO}_{3}$ and $\mathrm{TiO}_{2}$," Chemical Physics Letters, Vol. 69, No. 2, 1980, pp. 240-245. http://dx.doi.org/10.1016/0009-2614(80)85055-X 